Pesq. Vet. Bras. 35(5):451-455, maio 2015 DOI: $10.1590 / \mathrm{S} 0100-736 \mathrm{X} 2015000500011$

\title{
Ganho de peso, consumo de ração e histologia de órgãos de leitões alimentados com rações contendo baixos níveis de fumonisina $B_{1}{ }^{1}$
}

\author{
Pollyana C.M.C. Souto ${ }^{2}$, Leandra N.Z. Ramalho ${ }^{3}$, Fernando S. Ramalho ${ }^{3}$, Mayra C. Gregorio ${ }^{2}$, \\ Keliani Bordin², Anne-Marie Cossalter ${ }^{4}$, Isabelle P. Oswald ${ }^{4}$ e Carlos A.F. Oliveira ${ }^{2 *}$
}

\begin{abstract}
Souto P.C.M.C., Ramalho L.N.Z., Ramalho F.S., Gregorio M.C., Bordin K., Cossalter A.M., Oswald I.P. \& Oliveira C.A.F. 2015 [Weight gain, feed consumption and histology of organs from piglets fed rations containing low levels of fumonisin $B_{1}$.] Ganho de peso, consumo de ração e histologia de órgãos de leitões alimentados com rações contendo baixos níveis de fumonisina $\mathrm{B}_{1}$. Pesquisa Veterinária Brasileira 35(5):451-455. Departamento de Engenharia de Alimentos, Faculdade de Zootecnia e Engenharia de Alimentos, Universidade de São Paulo, Av. Duque de Caxias Norte, 225, Pirassununga, SP 13635-900, Brazil. E-mail: carlosaf@usp.br

Fumonisin $\mathrm{B}_{1}\left(\mathrm{FB}_{1}\right)$ is a secondary metabolite produced mainly by Fusarium verticilioides in several types of foods, particularly corn, which is the basis for composition of feed for several domestic animals. $\mathrm{FB}_{1}$ is particularly toxic to pigs, being the clinical manifestations evident in animals exposed to high concentrations of $\mathrm{FB}_{1}$ in the diet (generally above $30 \mathrm{mg} / \mathrm{kg}$ ). However, there are few studies on the effects of $\mathrm{FB}_{1}$ on pigs fed rations containing low concentrations of fumonisin, which are most probably found under field conditions. The aim of the study was to evaluate the effects of a 28-day exposure of piglets to low levels of $\mathrm{FB}_{1}$ in the feed on the weight gain, feed consumption, organ weights and histological aspects of the spleen, liver, lungs, kidneys and heart. Twenty-four pigs were assigned into 4 experimental groups and fed diets containing $0 \mathrm{mg}$ (control), $3.0 \mathrm{mg}, 6.0 \mathrm{mg}$ or $9.0 \mathrm{mg} \mathrm{FB}_{1} / \mathrm{kg}$ diet. The different diets did not affect $(\mathrm{P}>0.05)$ the weight gain or the weight of organs examined. There were no macroscopic or histological lesions in the spleen, liver, kidneys and heart. However, histological lesions were found in the lungs from all animals fed rations containing fumonisin, hence indicating that none of the $\mathrm{FB}_{1}$ levels used in the experiment could be considered as safe for piglets. Further studies on the mechanisms of toxic action of $\mathrm{FB}_{1}$ in pigs are needed, particularly under conditions of prolonged exposure to low contamination levels in the diet.
\end{abstract}

INDEX TERMS: Fumonisin $\mathrm{B}_{1}, \mathrm{FB}_{1}$, low levels, swine, toxic effects, mycotoxicosis.

\footnotetext{
${ }^{1}$ Recebido em 8 de maio de 2015.

Aceito para publicação em 27 de maio de 2015.

${ }^{2}$ Departamento de Engenharia de Alimentos, Faculdade de Zootecnia eEngenharia de Alimentos, Universidade de São Paulo (FZEA/USP), Campus Pirassununga, Av. Duque de Caxias Norte 225, Pirassununga, SP 13635-900, Brasil. E-mails: pollyanasouto@gmail.com, mayra.carraro@gmail.com, kelianibordin@gmail.com; *Autor para correspondência: carlosaf@usp.br

${ }^{3}$ Departamento de Patologia, Faculdade de Medicina de Ribeirão Preto, Universidade de São Paulo (FMRP/USP), Campus Ribeirão Preto, Av. Bandeirantes 3900, Monte Alegre, Ribeirão Preto, SP 14049-900, Brasil. E-mails: Iramalho@fmrp.usp.br, framalho@fmrp.usp.br

${ }^{4}$ Institute National de la Recherche Agronomique (INRA), UR66, Laboratoire de Pharmacologie-Toxicologie, 180, Chemin de Tournefeuille, Toulouse, France. E-mails: anne-marie.cossalter@toulouse.inra.fr, ioswald@ toulouse.inra.fr
}

RESUMO.- A fumonisina $\mathrm{B}_{1}\left(\mathrm{FB}_{1}\right)$ é um metabólito secundário produzido principalmente por Fusarium verticilioides em diversos tipos de alimentos, principalmente o milho, o qual constitui a base para composição de rações para várias espécies de animais domésticos. $\mathrm{A} \mathrm{FB}_{1}$ é particularmente tóxica para suínos, cujas manifestações clínicas são evidentes em animais expostos a altas concentrações de $\mathrm{FB}_{1}$ na ração (em geral, acima de $30 \mathrm{mg} / \mathrm{kg}$ ). No entanto, são escassos os estudos sobre os efeitos da $\mathrm{FB}_{1}$ em suínos alimentados com rações contendo baixas concentrações de fumonisinas, as quais são mais prováveis de serem encontradas em condições de campo. 0 objetivo do estudo foi avaliar os efeitos da exposição de leitões a baixos níveis de $\mathrm{FB}_{1}$ na ra- 
ção, durante 28 dias, sobre o ganho de peso, consumo de ração, peso relativo de órgãos e aspectos histológicos do baço, fígado, pulmões, rins e coração. Vinte e quatro leitões foram distribuídos em 4 grupos experimentais e alimentados com rações contendo $0 \mathrm{mg}$ (controle), 3,0mg, 6,0 mg ou 9,0mg $\mathrm{FB}_{1} / \mathrm{kg}$ de ração. As diferentes dietas não afetaram $(\mathrm{P}>0,05)$ o ganho de peso e nem o peso relativo dos órgãos analisados. Não foram constatadas lesões macroscópicas ou histopatológicas no baço, fígado, rins e coração. No entanto, foram observadas lesões histopatológicas nos pulmões de todos os suínos alimentados com rações contaminadas com fumonisinas, indicando que nenhum dos níveis de $\mathrm{FB}_{1}$ usados no experimento poderia ser considerado como seguro para suínos. São necessários novos estudos sobre os mecanismos de ação tóxica da $\mathrm{FB}_{1}$ em suínos, sobretudo em condições de exposição prolongada a baixos níveis de contaminação na ração.

TERMOS DE INDEXAÇÃO: Fumonisina $\mathrm{B}_{1}, \mathrm{FB}_{1}$, níveis baixos, suínos, efeitos tóxicos, micotoxicoses.

\section{INTRODUÇÃO}

Os fungos filamentosos produzem uma grande diversidade de metabólitos secundários, como pigmentos, antibióticos, fitotoxinas além de compostos tóxicos, denominados micotoxinas (Diaz 2005). Os fungos produtores de micotoxinas predominam em regiões com climas tropicais e subtropicais, tendo em vista que o desenvolvimento fúngico é favorecido pelas condições ambientais, principalmente pela temperatura e umidade, em substratos variados (Dilkin 2002). Cereais e sementes oleaginosas são frequentemente afetadas por micotoxinas durante a colheita, armazenamento e processamento, ocasionando perdas econômicas devido à contaminação de produtos agrícolas e a perda de produtividade animal (Sabino 1996).

Entre as micotoxinas mais frequentemente encontradas no Brasil, destacam-se as fumonisinas $\mathrm{B}_{1}\left(\mathrm{FB}_{1}\right), \mathrm{B}_{2}\left(\mathrm{FB}_{2}\right)$ e $\mathrm{B}_{3}\left(\mathrm{FB}_{3}\right)$, as quais são produzidas principalmente por Fusarium verticilioides e Fusarium proliferatum (Diaz 2005). Estas micotoxinas ocorrem fundamentalmente no milho e em alimentos à base de milho, sendo a $\mathrm{FB}_{1}$ a mais a mais tóxica, representando $70-80 \%$ do total de fumonisinas produzidas em culturas de laboratório ou em milho naturalmente contaminado (Cawood et al. 1991, Binder et al. 2007). A contaminação de matérias primas alimentares e rações com fumonisinas, tem sido associada a várias doenças que podem afetar a saúde de animais domésticos, sendo extremamente tóxicas para equídeos e suínos (Soriano, González \& Catalá 2005). Em casos de intoxicação aguda com elevados níveis da toxina, os animais apresentam um quadro caracterizado pela redução da eficiência reprodutiva, piora na conversão alimentar, diminuição da taxa de crescimento e do ganho de peso (Dilkin 2002). Como medida preventiva para evitar os efeitos das fumonisinas nas criações animais, os Estados Unidos e a União Europeia recomendam os limites de tolerância de 20,0mg/kg (milho em grão para rações de suínos) e $5,0 \mathrm{mg} / \mathrm{kg}$ (rações de suínos), respectivamente (Oliveira et al. 2014). Contudo, não há limites de tolerância para fumonisinas em ingredientes ou rações animais no Brasil.

Os suínos estão potencialmente expostos a níveis eleva- dos $\mathrm{FB}_{1}$ na ração em curto prazo, sendo o edema pulmonar suíno (EPS) a principal patologia induzida por esta micotoxina (Haschek et al. 2001). 0 principal mecanismo de ação tóxica da $\mathrm{FB}_{1}$ está relacionado com a inibição da biossíntese de esfingolipídeos de membrana celular (Soriano, González \& Catalá 2005). As manifestações clínicas decorrentes da intoxicação por altas concentrações de $\mathrm{FB}_{1}$ (em geral, acima de $30 \mathrm{mg} / \mathrm{kg}$ ) em suínos são bem descritas na literatura (Voss et al. 2001). No entanto, são escassos os estudos sobre os efeitos da exposição de suínos a rações contendo baixas concentrações de fumonisinas, as quais são mais prováveis de serem encontradas em condições de campo. Zomborszky-Kovacs et al. (2002) observaram um aumento significativo e dose-dependente nos pesos dos pulmões de suínos expostos a níveis de 5,0 a 10,0mg de $\mathrm{FB}_{1} / \mathrm{kg}$ de ração, porém não foram encontrados dados sobre os efeitos da $\mathrm{FB}_{1}$ em níveis menores que $5,0 \mathrm{mg} / \mathrm{kg}$. 0 objetivo do presente trabalho foi avaliar o ganho de peso, consumo de ração e o aspecto histológico de órgãos de leitões alimentados com rações contendo 3,0, 6,0 e 9,0mg de $\mathrm{FB}_{1} / \mathrm{kg}$.

\section{MATERIAL E MÉTODOS}

O estudo foi realizado no Institute National de la Recherche Agronomique/INRA, em Toulouse, França. Todos os procedimentos de experimentação em animais foram realizados conforme com as diretrizes europeias para o uso de animais para fins de investigação científica (Processo no TOXCOM/0018/PP IO, Comité d'Ethique de Farmacologie - Toxicologie de Toulouse - Midi Pyrénées, Toxcométhique, INRA). Vinte e quatro leitões desmamados de 4 semanas de idade, machos castrados (Pietrain/Duroc/Large-white) foram utilizados no presente estudo. Os leitões foram aclimatizados durante 1 semana no biotério do Laboratório ToxiAlim INRA (Toulouse, França) antes de serem utilizados para os protocolos experimentais. Após este período, os animais foram mantidos em baias por 28 dias, sendo 3 animais/baia. Água e ração foram fornecidos ad libitum durante todo o período experimental. Seis animais foram distribuídos para cada tratamento com base no peso corporal, observados diariamente e pesados semanalmente.

Os animais foram submetidos a quatro diferentes dietas, contaminadas com os seguintes níveis de fumonisina $\mathrm{B}_{1}\left(\mathrm{FB}_{1}\right) \mathrm{du}-$ rante 28 dias: dieta controle $\left(0 \mathrm{mg} \mathrm{FB}_{1} / \mathrm{kg}\right.$ de ração); 3,0mg FB $\mathrm{m}_{1}$ $\mathrm{kg}$ de ração, 6,0mg $\mathrm{FB}_{1} / \mathrm{kg}$ de ração e $9,0 \mathrm{mg} \mathrm{FB}_{1} / \mathrm{kg}$ de ração. A $\mathrm{FB}_{1}$ utilizada no experimento foi produzida no Veterinary Medical Diagnostic Laboratory da Universidade de Missouri, em Columbia, Estados Unidos, a partir do cultivo de cepas toxigênicas de Fusarium verticillioides, de acordo com Ogido et al. (2004). Os procedimentos para a produção de fumonisinas foram relatados previamente (Weibking et al. 1993).

As dietas foram fabricadas nas instalações do INRA em Rennes (França) e formuladas de acordo com as exigências nutricionais para leitões, como descrito por Grenier et al. (2011). Os extratos contendo a toxina foram misturados em suplementos de vitaminas e minerais, e, em seguida, incorporados na mistura dos cereais antes da granulação. Os níveis $\mathrm{FB}_{1}$ nas rações, bem como a ausência de concentrações detectáveis de outras micotoxinas, foram confirmados usando o método multi-micotoxina LC-MS/MS (de acordo com o protocolo de 365-007, GIP LABOCEA, França Relatório de análise 113074005). No 3o e 10ํ dia do experimento, todos os leitões foram imunizados por via subcutânea com Stellamune ${ }^{\circledR}$ Mycoplasma us.vet. (Pfizer Animal Health SA, France), de acordo com as especificações do fabricante. Esta imunização foi necessária para garantir a saúde dos animais e prevalecer apenas o efeito tóxico da $\mathrm{FB}_{1}$. 
As variáveis estudadas durante o período de intoxicação foram o ganho de peso e o consumo alimentar. No final do período experimental, os suínos foram insensibilizados por eletronarcose com posterior sangria, de acordo com os procedimentos padrão do INRA/Toulouse. Após, foram submetidos à necropsia e avaliação dos pesos do baço, fígado, pulmões, rins e coração. Amostras desses órgãos, de todos os animais dos 4 tratamentos, foram coletadas e fixadas em formol tamponado a 10\% para análise histopatológica. Os cortes de tecidos (pulmão, fígado, coração, rins e esôfago) foram desidratados por meio de álcoois graduados, embebidos em cera de parafina e cortados em espessura de $5 \mu \mathrm{m}$. Em seguida, estes cortes foram corados com hematoxilina-eosina (HE) para realização do exame histopatológico. Um patologista que desconhecia os tratamentos de intoxicação por $\mathrm{FB}_{1}$, sob a microscopia de luz, avaliou o grau de inflamação ou os danos celulares. A inflamação do tecido foi classificada da seguinte forma: grau 0 , mínima ou nenhuma evidência de inflamação; grau +, inflamação leve; grau ++, inflamação moderada a grave; grau +++ , inflamação grave. Os dados foram submetidos à ANOVA unidirecional, seguido de teste de Tukey, para analisar as diferenças entre as médias dos diferentes tratamentos. Valores de $\mathrm{P}<0,05$ foram considerados significativos.

\section{RESULTADOS}

A ingestão das rações experimentalmente contaminadas com fumonisina $\mathrm{B}_{1}\left(\mathrm{FB}_{1}\right)$ não prejudicou $(\mathrm{P}>0,05)$ o desempenho dos animais (Quadro 1), e também não afetou $(P>0,05)$ os pesos relativos dos órgãos, em comparação com o grupo controle (Quadro 2). Nenhum sinal clínico de intoxicação foi observado em qualquer tratamento durante o experimento. Durante a necropsia, não foram encontradas alterações macroscópicas no pulmão, rim, coração e fígado. Microscopicamente, apenas nos pulmões foi ob- servada uma inflamação intersticial (de moderada a grave), sem edema alveolar. Os suínos alimentados com as rações contaminadas com 3,0 ou $6,0 \mathrm{mg} \mathrm{FB}_{1} / \mathrm{kg}$ de ração, apresentaram lesões pulmonares classificadas como leves $(+)$ ou moderadas (++) (Fig.1), sendo que lesões moderadas à

Quadro 1. Peso corporal, ganho de peso e consumo de ração de leitões alimentados com rações controle e rações contaminadas com fumonisina $B_{1}$ após 28 dias de intoxicação*

\begin{tabular}{ccccc}
\hline $\begin{array}{c}\text { Fumonisina } B_{1} \\
\text { na ração }(\mathrm{mg} / \mathrm{kg})\end{array}$ & $\begin{array}{c}\text { Peso inicial } \\
(\mathrm{kg})\end{array}$ & $\begin{array}{c}\text { Peso final } \\
(\mathrm{kg})\end{array}$ & $\begin{array}{c}\text { Ganho de peso } \\
(\mathrm{kg} / \text { semana) }\end{array}$ & $\begin{array}{c}\text { Consumo de ra- } \\
\text { ção }(\mathrm{kg} / \text { semana) }\end{array}$ \\
\hline 0 (Controle) & $10,4 \pm 0,6$ & $28,4 \pm 2,0$ & $4,5 \pm 0,8$ & $8,0 \pm 0,8$ \\
3 & $10,5 \pm 0,9$ & $30,2 \pm 2,7$ & $4,9 \pm 0,9$ & $7,6 \pm 1,2$ \\
6 & $11,1 \pm 0,4$ & $32,0 \pm 1,3$ & $5,2 \pm 0,8$ & $8,2 \pm 1,3$ \\
9 & $10,8 \pm 0,9$ & $30,6 \pm 1,4$ & $4,9 \pm 0,9$ & $7,6 \pm 1,4$
\end{tabular}

Em uma coluna, não foram encontradas diferenças entre as médias $(\mathrm{P}>0,05) . *$ Valores se referem à média \pm desvio padrão para 6 animais em cada tratamento.

Quadro 2. Pesos relativos de órgãos de leitões alimentados com rações controle e rações contaminadas com fumonisina $B_{1}$ após 28 dias de intoxicação*

\begin{tabular}{cccccc}
\hline Fumonisina $\mathrm{B}_{1}$ & \multicolumn{5}{c}{ Pesos relativos (g/100g de peso corpóreo) } \\
\cline { 2 - 6 } na ração $(\mathrm{mg} / \mathrm{kg})$ & Baço & Fígado & Pulmões & Rins & Coração \\
\hline 0 (Controle) & $0,24 \pm 0,02$ & $3,37 \pm 0,24$ & $1,33 \pm 0,30$ & $0,33 \pm 0,03$ & $0,76 \pm 0,20$ \\
3 & $0,26 \pm 0,06$ & $3,08 \pm 0,33$ & $1,32 \pm 0,40$ & $0,31 \pm 0,08$ & $0,72 \pm 0,30$ \\
6 & $0,22 \pm 0,04$ & $3,24 \pm 0,37$ & $1,10 \pm 0,140$ & $0,29 \pm 0,10$ & $0,64 \pm 0,07$ \\
9 & $0,20 \pm 0,02$ & $3,11 \pm 0,40$ & $1,24 \pm 0,20$ & $0,33 \pm 0,03$ & $0,58 \pm 0,06$
\end{tabular}

Em uma coluna, não foram encontradas diferenças entre as médias $(\mathrm{P}>0,05)$. * Valores se referem à média \pm desvio padrão para 6 animais em cada tratamento.

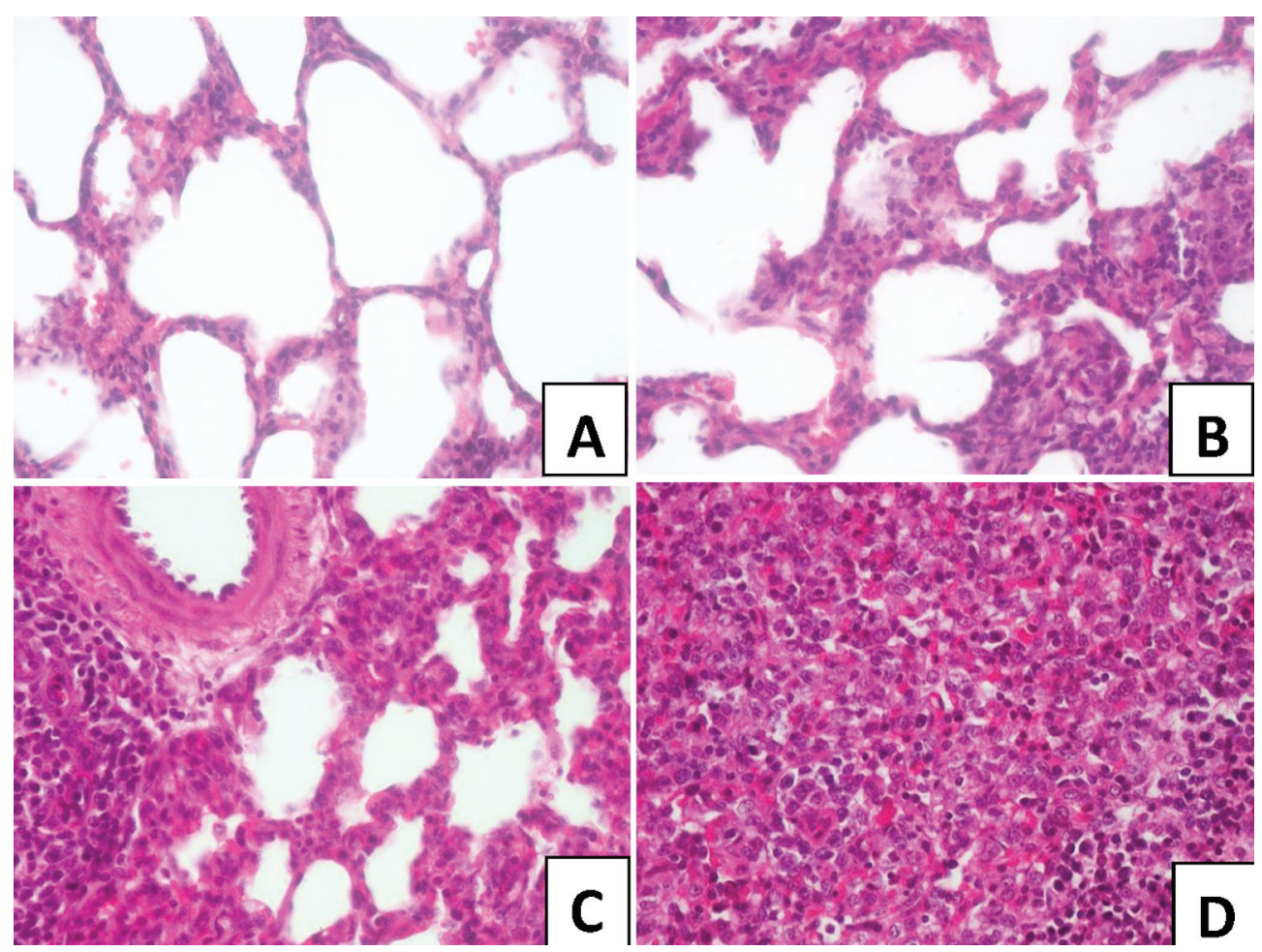

Fig.1. Corte histológico de pulmão demonstrando lesões causadas pela fumonisina $\mathrm{B}_{1}\left(\mathrm{FB}_{1}\right)$ em leitões alimentados com ração controle $0 \mathrm{mg} \mathrm{FB} / \mathrm{kg}$, ou rações contaminadas com 3,0, 6,0, ou 9,0 $\mathrm{mg} \mathrm{FB}_{1} / \mathrm{kg}$. As lesões foram estabelecidas após exames histopatológicos de acordo com o grau das lesões. (A) Zero (sem lesões). (B) Lesão leve (+), (C) Lesão moderada (++), (D) Lesão grave (+++). HE, obj.40x. 
grave (+++) foram observadas nos animais que consumiram rações com $8,0 \mathrm{mg} \mathrm{FB}_{1} / \mathrm{kg}$. Todos os animais do grupo controle apresentaram pulmões classificados como grau 0 (mínima ou nenhuma evidência de inflamação). Os outros órgãos analisados, como esôfago, rim, coração e fígado, não apresentaram lesões histopatológicas significantes em todos os tratamentos.

\section{DISCUSSÃO}

No presente estudo, os leitões foram expostos a níveis baixos de fumonisinas, normalmente encontrados em uma variedade de substratos em condições de campo, principalmente o milho (base da ração para animais). A literatura demonstra que grande parte dos dados sobre os efeitos das fumonisinas em animais domésticos, incluindo aves, cavalos e suínos, foi obtida utilizando rações mono-contaminada com altas concentrações de $\mathrm{FB}_{1}$ (Dilkin et al. 2003, Butkeraitis et al. 2004, Direito et al. 2009). Os dados deste estudo procurou determinar os efeitos da ingestão sub-crônica de rações contaminadas com níveis baixos de $\mathrm{FB}_{1}$ no desempenho animal e na histopatologia, visto que os suínos são altamente susceptíveis e estão potencialmente expostos a níveis elevados de $\mathrm{FB}_{1}$. Assim, sob o ponto de vista de segurança alimentar, são de extrema importância os estudos que utilizam níveis menores da micotoxina para determinar os limites toleráveis que não sejam prejudiciais para o organismo animal.

A fumonisina $\mathrm{B}_{1}\left(\mathrm{FB}_{1}\right)$ não interferiu no consumo médio de ração e no ganho de peso corporal. Considerando-se os baixos níveis de contaminação utilizados, nossos dados não são surpreendentes e corroboram os estudos que utilizaram rações contaminadas com $10,0 \mathrm{mg} \mathrm{FB} / 1 \mathrm{~kg}$ (Dilkin et al. 2003) ou 6,0mg FB $1 / \mathrm{kg}$ (Grenier et al. 2011) sobre o desempenho de suínos, os quais não relataram nenhum efeito sobre o ganho de peso corporal. Leitões alimentados com rações que continham de 1,0-10,0 $\mathrm{FB}_{1} \mathrm{mg} / \mathrm{kg}$ durante 4-8 semanas, também não apresentaram efeitos tóxicos das fumonisinas (Zomborszky et al. 2000, 2002, Dilkin et al. 2010). Burel et al. (2013) demonstraram que a exposição a uma concentração moderada de fumonisinas $(11,8 \mathrm{ppm})$ não teve efeito sobre o desempenho dos animais (consumo de ração, crescimento e eficiência alimentar). Porém, os autores observaram que a $\mathrm{FB}_{1}$ alterou a razão de esfingolipídeos de membrana, embora nenhuma mortalidade ou patologia tenha sido constatada nos leitões ao longo de 63 dias de exposição.

Alterações no peso de órgãos de suínos intoxicados com $\mathrm{FB}_{1}$ foram previamente observadas por Harvey et al. (1995). Os autores relataram que o peso do fígado foi reduzido, ocorrendo um aumento de peso do pulmão de leitões intoxicados com níveis elevados da micotoxina $\left(100 \mathrm{mg} \mathrm{FB}_{1} / \mathrm{kg}\right.$ de ração) durante 35 dias. No presente estudo, a exposição dos animais a níveis baixos de $\mathrm{FB}_{1}$ por 28 dias não resultou em alterações nos pesos de órgãos em todos os tratamentos. Dilkin et al. (2003) também não encontraram diferenças significativas no peso médio do coração, fígado e pulmões, ao avaliarem o efeito de $10,0 \mathrm{mg} \mathrm{FB}_{1} / \mathrm{kg}$ e de $30,0 \mathrm{mg}$ $\mathrm{FB}_{1} / \mathrm{kg}$ durante 28 dias de exposição. Em contrapartida, suínos expostos a níveis de 5,0 a 10,0 $\mathrm{mg} \mathrm{FB}_{1} / \mathrm{kg}$ de ração, a ingestão da toxina induziu um aumento significativo dose-dependente nos pesos dos pulmões de suínos (Zomborszky et al. 2002). Estas alterações podem ser causadas por diferenças nas condições experimentais, no estado de saúde dos animais ou interações com outros contaminantes. A principal micotoxicose induzida por fumonisinas em suínos é caracterizada por lesão pulmonar, conhecida como edema pulmonar suíno (EPS). Dependendo da dose ingerida, a $\mathrm{FB}_{1}$ pode causar danos hepáticos, cardiovasculares e imunossupressão, devido a alterações no metabolismo dos esfingolipídeos (Haschek et al. 2001). Apesar da ausência de efeitos da $\mathrm{FB}_{1}$ no desempenho animal, observou-se danos pulmonares, apresentando infiltrado celular inflamatório intersticial classificado como leve a moderado, em animais que consumiram rações contaminadas com $3,0 \mathrm{mg} \mathrm{FB} / \mathrm{kg}$ de ração, lesão moderada para o nível de 6,0mg $\mathrm{FB}_{1} / \mathrm{kg}$ e lesão de moderada a grave para a ingestão do nível de $9,0 \mathrm{mg}$ $\mathrm{FB}_{1} / \mathrm{kg}$ de ração. Embora no presente trabalho tenham sido constatadas alterações histopatológicas nos pulmões dos leitões de todos os tratamentos, não foi observado edema pulmonar. Nos estudos de Grenier et al. (2011), as lesões pulmonares microscópicas foram observadas sem sinais macroscópicos, os quais estão de acordo com os dados do presente estudo. Os danos pulmonares induzidos pela $\mathrm{FB}_{1}$ no nível de 6,0 FB $\mathrm{mg} / \mathrm{kg}$ de ração, foram relacionados com alterações do tecido linfóide bronquiolar associados a danos vasculares Grenier et al. (2011). Contudo, os dados deste estudo não estão de acordo com Zomborszky et al. (2000), o qual observaram a presença de edema pulmonar em suínos alimentados com ração contaminada com 5,0mg FB $/ 1 \mathrm{~kg}$. Ainda, Dilkin et al. (2003) descreveram o edema pulmonar durante a exposição de leitões recém-desmamados submetidos a $10,0 \mathrm{mg} \mathrm{FB} / \mathrm{kg}$ na ração.

A ausência de lesões histopatológicas de outros órgãos avaliados (esôfago, rins, coração e fígado) é coerente com as baixas concentrações de $\mathrm{FB}_{1}$ nas rações utilizadas no presente estudo. Os efeitos tóxicos da $\mathrm{FB}_{1}$ no fígado, por exemplo, têm sido relatados em vários trabalhos utilizando materiais altamente contaminados (Voss et al. 2001). Haschek et al. (2001) demonstraram que alterações morfológicas foram observadas após a ingestão de $23 \mathrm{mg} / \mathrm{kg}$ ou após administração intravenosa de $\mathrm{FB}_{1}$ em suínos. Por outro lado, danos no fígado ocorreram após a ingestão de um nível menor que $12 \mathrm{ppm}$ de fumonisinas (Motelin et al. 1994). Mesmo com a exposição de suínos submetidos a 6,0mg $\mathrm{FB}_{1} / \mathrm{kg}$ ter induzido lesões hepáticas (Grenier et al. 2011), os suínos que receberam ração contaminada com 9,0 $\mathrm{mg} \mathrm{FB}_{1} / \mathrm{kg}$ (maior nível de $\mathrm{FB}_{1}$ no presente estudo), não apresentaram lesões histopatológicas no fígado. Ainda, não foram encontradas alterações no rim ou coração, nem qualquer sinal de inflamação nos animais alimentados com 3,0, 6,0 ou $9,0 \mathrm{mg} \mathrm{FB} / \mathrm{kg}$ de ração.

\section{CONCLUSÕES}

As concentrações de $3,0-9,0 \mathrm{mg} / \mathrm{kg}$ de fumonisina $\mathrm{B}_{1}$ $\left(\mathrm{FB}_{1}\right)$ utilizadas neste estudo não induziram manifestações clínicas evidentes e nem alteraram o ganho de peso, consumo de ração ou alterações macroscópicas em órgãos dos animais expostos às rações contaminadas. 
No entanto, lesões histopatológicas nos pulmões foram constatadas de maneira dose-dependente, indicando que nenhum dos níveis de $\mathrm{FB}_{1}$ usados no experimento poderia ser considerado como seguro para suínos.

São necessários novos estudos sobre os mecanismos de ação tóxica da $\mathrm{FB}_{1}$ em suínos, sobretudo em condições de exposição prolongada a baixos níveis de contaminação na ração.

Agradecimentos.- Os autores agradecem à Coordenação de Aperfeiçoamento de Nível Superior (CAPES/PDSE) - processo BEX no 5780/13-6, pela bolsa concedida.

\section{REFERÊNCIAS}

Binder E.M., Tan L.M., Chin L.J., Handl J. \& Ichard J. 2007. Worldwide occurrence of mycotoxins in commodities, feeds and feed ingredients. Anim. Feed Sci. Technol. 137(3/4):265-282.

Burel C., Tanguy M., Guerre P., Boilletot E., Cariolet R., Queguiner M., Postollec G., Pinton P., Salvat G., Oswald I.P. \& Fravalo P. 2013. Effect of low dose of fumonisins on pig health: immune status, intestinal microbiota and sensitivity to Salmonella. Toxins 5(4):841-864.

Butkeraitis P., Oliveira C.A.F., Ledoux D.R., Ogido R., Albuquerque R., Rosmaninho J.F. \& Rottinghaus G.E. 2004. Effect of dietary fumonisin $B_{1}$ on laying Japanese quail. Brit. Poult. Sci. 45(6):798-801.

Cawood M.E., Gelderblom W.C.A., Vleggaar R., Behrend Y., Thiel P.G. \& Marasas W.F.O. 1991. Isolation of the fumonisin mycotoxins - a quantitative approach. J. Agric. Food Chem. 39(11):1958-1962.

Diaz D.E. 2005. The mycotoxin blue book. Nottingham University Press, Nottingham, UK. 349p.

Dilkin P. 2002. Micotoxicose suína: aspectos preventivos, clínicos e patológicos. Biológico, São Paulo, 64(2):187-191.

Dilkin P., Zorzete P., Mallmann C.A., Gomes J.D., Utiyama C.E., Oetting L.L. \& Corrêa B. 2003. Toxicological effects of chronic low doses of aflatoxin $B_{1}$ and fumonisin $\mathrm{B}_{1}$-containing Fusarium moniliforme culture material in weaned piglets. Food Chem. Toxicol. 41(10):1345-1353.

Dilkin P., Direito G., Simas M.M., Mallmann C.A. \& Corrêa B. 2010. Toxicokinetics and toxicological effects of single oral dose of fumonisin $\mathrm{B}_{1}$ containing Fusarium verticillioides culture material in weaned piglets. Chem. Biol. Interact. 185(3):157-162.

Direito G.M., Almeida A.P., Aquino S., Reis T.A., Pozzi C.R. \& Corrêa B. 2009. Evaluation of sphingolipids in Wistar rats treated to prolonged and single oral doses of fumonisin $B_{1}$. Int. J. Mol. Sci. 10(1):50-61.
Grenier B., Loureiro-Bracarense A.P., Lucioli J., Pacheco G.D., Cossalter A.M., Moll W.D., Schatzmayr G. \& Oswald I.P. 2011. Individual and combined effects of subclinical doses of deoxynivalenol and fumonisins in piglets. Mol. Nutr. Food Res. 55(5):761-771.

Harvey R.B., Edrington T.S., Kubena L.F., Elissalde M.H. \& Rottinghaus G.E. 1995. Influence of aflatoxin and fumonisin B1- containing culture material on growing barrows. Am. J. Vet. Res. 56(12):1668-1672.

Haschek W.M., Gumprecht L.A., Smith G., Tumbleson M.E. \& Constable P.D. 2001. Fumonisin toxicosis in swine: An overview of porcine pulmonary edema and current perspectives. Environ. Health Perspect. 109(Suppl.2): 251-257.

Motelin G.K., Haschek W.M., Ness D.K., Hall W.F., Harlin K.S., Schaeffer D.J. \& Beasley V.R. 1994. Temporal and dose-response features in swine fed corn screenings contaminated with fumonisin mycotoxins. Mycopathologia 126(1):27-40.

Ogido R., Oliveira C.A.F., Ledoux D.R., Rottinghaus G.E., Corrêa B., Butkeraitis P., Reis T.A., Gonçales E. \& Albuquerque R. 2004. Effects of prolonged administration of aflatoxin $\mathrm{B}_{1}$ and fumonisin $\mathrm{B}_{1}$ in laying Japanese quail. Poult. Sci. 83(12):1953-1958.

Oliveira C.A.F., Corassin C.H., Corrêa B. \& Oswald I.P. 2014. Animal Health: mycotoxins, p.358-377. In: Alfen N.V. (Ed.), Encyclopedia of Agriculture and Food Systems. Vol.1. Elsevier, San Diego.

Sabino M. 1996. Micotoxinas em Alimentos, p.461-472. In: Oga S. (Ed.), Fundamentos de Toxicologia. Atheneu Editora, São Paulo.

Soriano J.M., González L. \& Catalá A.I. 2005. Mechanism of action of sphingolipids and their metabolites in the toxicity of fumonisin $\mathrm{B}_{1}$. Progr. Lipids Res. 44(6):345-356.

Voss K.A., Riley R.T., Norred W.P., Bacon C.W., Meredith F.I., Howard P.C., Plattner R.D., Collins T.F.X., Hansen D.K. \& Porter J.K. 2001. An overview of rodent toxicities: liver and kidney effects of fumonisins and Fusarium moniliforme. Environ. Health Perspect. 109(Suppl.2):259-266.

Weibking T.S., Ledoux D.R., Bermudez A.J., Turk J.R. \& Rottinghaus G.E. 1993. Effects of feeding Fusarium moniliforme culture material, containing known levels of fumonisin $B_{1}$, on the young broiler chick. Poult. Sci. 72(3):456-466.

Zomborszky M.K., Vetési F., Repa I., Kovacs F., Bata A., Horn P. Tóth A. \& Romvari R. 2000. Experiment to determine limits of tolerance for fumonisin B1 in weaned piglets. J. Vet. Med. B 47(4):277-286.

Zomborszky-Kovács M., Kovács F., Horn P., Vetési F., Repa I., Tornyos G. \& Tóth A. 2002. Investigations into the time - and dose-dependent effect of fumonisin B1 in order to determine tolerable limit values in pigs. Livest. Prod. Sci. 76(3):251-256. 